Revista de Investigación en Psicología. Vol. 2, № 1. pp. 91 - 102

\title{
LA VIOLENCIA JUVENIL EN LIMA METROPOLITANA ${ }^{1}$
}

\section{Carlos Velásquez Centeno}

Investigación descriptivo-comparativa orientada a identificar la existencia de diferencias en la percepción de la violencia entre los grupos de alumnos que han participado vs el grupo de alumnos que no han participado en actos violentos de Lima Metropolitana. Se elaboró y se obtuvo la validez y confiabilidad de la Escala de la Percepción de la Violencia Juvenil, la cual presenta tres sub-escalas: Autojustificación, Percepción del Control Parental y Tendencia sociopática. Se aplicó a una muestra intencional de 1132 alumnos de Centros Educativos Nacionales de $3^{\circ}$ al $5^{\circ}$ grado de secundaría de los diferentes conos. Los resultados encontrados permiten determinar que el grupo de participación en actos violentos tiene una percepción acorde con su condición, expresándose en la autojustificación de sus actos y su tendencia a desarrollar conductas sociopáticas. Del mismo modo el grupo de participación percibe que sus padres tienen un mayor control sobre ellos que los de no-participación. Los varones del grupo de participación se encuentran más involucrados en actos violentos que las mujeres, en comparación con los del grupo de no-participación.

Palabras clave: Violencia estudiantil, violencia en Lima Metropolitana, percepción y violencia, actitud y violencia.

Comparative-descriptive investigation guided to identify the existence of differences in the perception of the violence between the groups of students that have participated against the group of students that have not participated in violent acts in Metropolitan Lima. It was elaborated and is obtained the validity and reliability of the Scale of Perception of the Juvenile Violence, which presents three sub-scales:

Self-justification, Perception of the Parental Control and Sociopatic Trends. It was applied to an intentional sample of 1132 pupils of National Educational Centers of 30 to the 50 year, of high school of the different canes of the city. The results found permit to determine that the group of participation in violent acts has an agreed perception with their condition, being expressed in the selj-justification of their acts and their tendency to develop sociopatic conducts. In the same way the parents of the group of participation have a greater control over their children, that those of not-participation. The males of the group of participation are found more involved in violent acts that the women, in comparison with those of non-participation.

Key words: Students violence, violence in Metropolitan Lima, perception and violence, attitude and violence.

1 Miembros: C. Arenas, M. Campos, V. Montero. R. Elizalde y W. Guevara. 
La violencia padecida y ejercida por los escolares recorre día a día calles, plazas públicas, hogares, escuelas, espectáculos deportivos y artísticos, es decir, es un fenómeno sumamente complejo de múltiples dimensiones. Para entender el fenómeno debemos ubicarlo en el marco social y nacional en el que se desarrolla, reconocer las dinámicas que lo generan, mantienen, reproducen o permiten su modificación. Es necesario contar con enfoques integrales que permitan visualizar la compleja multicausalidad y las magnitudes del fenómeno.

Del mismo modo se hace necesario explicar la percepción del fenómeno desde el punto de vista adolescente y juvenil no sólo desde el del adulto (perspectiva basada en el control y la coacción, paternalismo-represivo y asistencialismo).

Para comprender la violencia y las actitudes como un factor motivacional del comportamiento, se requiere partir de su relación con la agresión. La agresión es un fenómeno conductual. Es una conducta hostil o destructiva que expresa una tendencia o intención dirigida a producir daño físico o psicológico a otra u otras personas (Morales, 1994).

La violencia es un fenómeno social. Es la instauración del uso de la agresión como medio para la consecución de los objetivos deseados. Esto se asocia a una clasificación que distingue entre agresión hostil y agresión instrumental (Myers, 1995). La primera es impulsada por el enojo, y la segunda se dirige también a producir daño, pero sólo como medio para lograr un fin deseado (Myers, 1995).

De la exposición y revisión conceptual asumida en la presente investigación pretendemos aportar a la psicología social experimental y en especial, a la investigación cuantitativa de las actitudes. No sin antes hacer una breve reseña histórica de los aportes previamente realizados.

La mayoría de los estudios sobre violencia en nuestro medio se enmarcan dentro de la investigación cualitativa: Moragues (1989), Tong (1995), García (1995), Trahtenberg y otros (1995), Orellana y García (199ú), Elizalde y cols. (1997), Cruzado (1997), Arenas (1998), Montgomery (1998), Martínez y Tong (1998), quienes describen, diagnostican y formulan soluciones de acuerdo a diferentes enfoques y criterios. Por otro lado desde un corte ecléctico, la 
psicología comunitaria representada por Vallejo y Montero (1990), desarrolla planes y aplicaciones prácticas al problema.

Por parte del Estado existen tentativas aisladas de tipo teórico y práctico que tratan de explicar y dar solución al problema, tal es el caso del Congreso de la República que ha creado varias comisiones técnicas y parlamentarias, que de manera coyuntural han tratado de conceptual izar el tema, e igualmente se ha apoyado el trabajo readaptativo y preventivo del INABIF así como, en el ámbito de los Centros Educativos con mayor incidencia de actos violentos en Lima y algunas ciudades del interior del país, se viene desarrollando el Programa de Prevención de la Violencia del Ministerio de Educación.

Por otro lado, se han desarrollado algunos estudios piloto realizados por Anicama (1989), Varona (1991), Masías (1994) y Masías y Anicama (1994), que han probado con éxito los efectos de Programas de Intervención sobre las habilidades sociales en alumnos con conductas agresivas.

Ante la carencia de investigaciones descriptivo-comparativas en nuestro medio, sobre las actitudes y percepciones de los alumnos ante la violencia juvenil, que nos permitan tener en el futuro indicadores para realizar Programas de Intervención, es que planteamos como objetivo de la presente investigación: Determinar las diferencias en la percepción y actitudes ante la violencia por parte de un grupo de alumnos de Centros Educativos Nacionales de los diferentes Conos de Lima Metropolitana, que hayan participado y no participado en actos violentos.

\section{MÉTODO}

\section{Sujetos}

La muestra intencional estuvo conformada por 1132 alumnos de ambos sexos de 20 Centros Educativos Nacionales de $3^{\circ}$ a $5^{\circ}$ grado de educación secundaría, de los cinco conos poblaciones de Lima Metropolitana, cuyas edades fluctuaron entre 13 a 18 años de edad, tal como figura en el cuadro 1: 


\section{Cuadro 1}

\section{Características de la muestra}

\begin{tabular}{|c|c|c|c|c|}
\hline \multirow{2}{*}{$\mathrm{CONO}$} & \multirow{2}{*}{ CENTRO EDUCATIVO } & \multicolumn{2}{|c|}{ SEXO } & \multirow{2}{*}{$\begin{array}{c}\text { TOTAL } \\
\text { POR } \\
\text { CONO }\end{array}$} \\
\hline & & MASCULINO & FEMENINO & \\
\hline \multirow{5}{*}{ NORTE } & Wiesse & 47 & 13 & \multirow{5}{*}{302} \\
\hline & Peruano Suizo & 31 & 29 & \\
\hline & Estados Unidos & 25 & 35 & \\
\hline & José Granda & 39 & 21 & \\
\hline & Pareja Paz Soldan & 62 & & \\
\hline \multirow{4}{*}{ ESTE } & Antenor Orrello & 47 & 08 & \multirow{4}{*}{185} \\
\hline & Francisco Bolognesi & 25 & 17 & \\
\hline & El Bosque & 27 & 20 & \\
\hline & Javier Heraud & 35 & 06 & \\
\hline \multirow{5}{*}{ SUR } & Ramiro Priale & 31 & 27 & \multirow{5}{*}{274} \\
\hline & San Juan & 39 & 25 & \\
\hline & J, C. Mariategui & 35 & 13 & \\
\hline & Inca Pachacutec & 37 & 21 & \\
\hline & Ricardo Palma & 46 & --- & \\
\hline \multirow{5}{*}{$\begin{array}{c}\text { LIMA } \\
\text { CIUDAD }\end{array}$} & Guadalupe & 60 & --- & \multirow{5}{*}{296} \\
\hline & Meliton Carbajal & 60 & --- & \\
\hline & Alfonso Ugarte & 58 & --- & \\
\hline & Mariano Melgar & 60 & --- & \\
\hline & Pedro A. Labarthe & 46 & 12 & \\
\hline \multirow{2}{*}{ CALLAO } & Dos De Mayo & 25 & 19 & \multirow{2}{*}{75} \\
\hline & Rep, De Venezuela & 31 & --- & \\
\hline & TOTAL GENERAL & 866 & 266 & 1132 \\
\hline
\end{tabular}

\section{Instrumento:}

Para cumplir con el objetivo de la investigación se elaboró y validó la "Escala de Percepción de la Violencia Juvenil" (EPEVIO). EPEVIO no es un test psicológico, sino más bien una escala actitudinal y de percepción social orientado a evaluar características básicas del comportamiento psicosocial del individuo, con respecto a la violencia juvenil.

\section{Objetivo:}

Contribuir a un conocimiento de los factores psicosociales implicados en el desencadenamiento de la violencia juvenil y en consecuencia la percepción que tienen de ella. 


\section{Estructura del instrumento:}

La Escala de Percepción de la Violencia Juvenil en su versión final está conformada por 30 Ítems seleccionados a través del análisis factorial, quedando agrupados de la siguiente manera:

17 Ítems evalúan la Autojustificación.

07 Ítems evalúan la Percepción del Control Parental; 05

ítems evalúan la Tendencia sociopática.

Validez y Con fiabilidad: Se realizó con una muestra de 107 alumnos de $4^{\circ}$ y $5^{\circ}$ de secundaria. (ANEXO 1)

\section{Procedimiento:}

La muestra estuvo conformada por alumnos de 20 Centros Educativos Nacionales de Lima Metropolitana, seleccionados por conocerse la existencia de actos de pandillaje, estableciéndose contacto a través de la Coordinación del Programa de Prevención de la violencia, de la Oficina de Coordinación Universitaria del Ministerio de Educación, quienes nos autorizaron la evaluación.

La administración de la EPEVIO a los alumnos de la muestra intencional se realizó estableciendo coordinaciones con los Psicólogos Promotores del "Programa de Prevención de Violencia" de cada Centro Educativo, quienes sobre la base del conocimiento de su realidad agruparon a los alumnos de $3^{\circ}$ al $5^{\circ}$ de secundaría en dos grupos de acuerdo a la variable participación o noparticipación en actos violentos.

\section{RESULTADOS}

$\mathrm{Al}$ comparar las medias de los grupos de alumnos que han y no han participado en actos violentos a través de la $t$ de student para grupos independientes, se obtuvo diferencias significativas a favor del grupo de alumnos que han participado en actos violentos en las escalas autojustificación, percepción del control parental y tendencia sociopática (Tabla 1). 


\section{Tabla 1}

Escalas de autojustificación, percepción del control parental y tendencia sociopatica entre los grupos que han y no han participado en actos violentos.

\begin{tabular}{|c|c|c|c|c|}
\hline \multirow{2}{*}{ GRUPOS } & \multicolumn{2}{|c|}{ ACTOS VIOLENTOS } & \multirow{2}{*}{ t } & P \\
\cline { 2 - 5 } ESCALAS & $\begin{array}{c}\text { PARTICIPACION } \\
\mathbf{n}=559\end{array}$ & $\begin{array}{c}\text { NO } \\
\text { PARTICIPACION } \\
\text { n=573 }\end{array}$ & & \\
\hline Autojustificación & 48.53 & 45.31 & -5.07 & $.000 *$ \\
\hline $\begin{array}{c}\text { Percepción del Control } \\
\text { Parental }\end{array}$ & 15.38 & 14.53 & -3.39 & $.001 *$ \\
\hline \begin{tabular}{c} 
Tendencia Sociopatica \\
\hline
\end{tabular} & 13.39 & 12.53 & -3.73 & $.000 *$ \\
\hline
\end{tabular}

(*) Significativo $\mathrm{p}<.05$

\section{Tabla 2}

Análisis de Varianza entre los conos, grupos y cono-grupo

\begin{tabular}{|c|l|c|l|}
\hline ESCALAS & \multicolumn{1}{|c|}{ VARIABLE } & F & P \\
\hline \multirow{3}{*}{ Autojustificación } & Cono & 2.00 & $\mathbf{0 9 2}$ \\
& Grupo & $26.27 *$ & $.000 *$ \\
& Cono-Grupo & 1.24 & .283 \\
\hline \multirow{2}{*}{ Percepción del Control } & Cono & $\mathbf{0 . 4 5}$ & .773 \\
Parental & Grupo & $11.31 *$ & $.001 *$ \\
& Cono-Grupo & $\mathbf{1 . 2 0}$ & .309 \\
\hline \multirow{3}{*}{ Tendencia Sociopatica } & Cono & .863 & .486 \\
& Grupo & $\mathbf{1 0 . 0 4} *$ & $\mathbf{. 0 0 0} *$ \\
& Cono-Grupo & $\mathbf{1 . 0 4}$ & .387 \\
\hline
\end{tabular}

(*) Significativo $p<.05$

Los resultados del análisis de varianza en las escalas de Autojustificación, percepción del control parental y tendencia sociopática para los conos, grupos y la interacción entre cono-grupo, nos muestra que las diferencias las marca el grupo en las tres escalas, obteniendo mayor media el grupo que ha participado en actos violentos (Tabla 2).

Como se puede observar en la tabla 3, en la Escala de autojustificación no hay diferencia en cada uno de los grupos. 
En el grupo de no-participación en actos violentos de la Escala de percepción del control parental, la diferencia la marca el cono de Lima Ciudad que alcanza una media mayor (15.36) que el cono sur que obtuvo la media menor (10.26).

Tabla 3

Análisis de varianza de las escalas de Autojustificación (I), percepción del control parental (II) y tendencia sociopática (III) para cada grupo independiente.

\begin{tabular}{|c|c|c|c|c|c|c|c|c|c|c|c|c|c|c|}
\hline GRUPOS & \multicolumn{7}{|c|}{ NO PARTICIPACION EN ACTOS VIOLENTOS } & \multicolumn{7}{|c|}{ PARTICIPACION EN ACTOS VIOLENTOS } \\
\hline CONOS & $\begin{array}{c}\text { NORT } \\
\mathrm{E}\end{array}$ & ESTE & SUR & $\begin{array}{l}\text { LIMA } \\
\text { CIUDAD }\end{array}$ & CALLAO & $\mathbf{F}$ & p & NORTE. & ESTE & SUR & $\begin{array}{c}\text { LIMA } \\
\text { CIUDAD }\end{array}$ & CALLAO & $\mathbf{F}$ & p \\
\hline $\begin{array}{l}\text { ESCALA } \\
\text { I }\end{array}$ & 45.76 & 45.30 & 44.51 & 45.46 & 45.42 & .24 & .913 & 47.93 & 47.40 & 48.71 & 49.25 & 49.92 & .78 & .530 \\
\hline $\begin{array}{c}\text { ESCALA } \\
\text { II }\end{array}$ & 14.88 & 14.30 & 10.26 & 1536 & 14.80 & 3.93 & $.003 *$ & 14.80 & 14.74 & 15.79 & 15.07 & 16.40 & 2.85 & $.023^{*}$ \\
\hline $\begin{array}{c}\text { ESCALA } \\
\text { III }\end{array}$ & 12.68 & 12.24 & 11.80 & 10.01 & 12.97 & 2.04 & .097 & 13.18 & 12.86 & 13.51 & 13.23 & 14.57 & 2.52 & .040 \\
\hline
\end{tabular}

(*) Significativo $\mathrm{p}<.05$

Por otro lado el grupo de participación en actos violentos, mostró en la Escala de percepción del control parental diferencias en sus medias, obteniendo mayor puntaje el Callao (16.40) sobre los Conos Norte (14.80) y Este (14.74).

Mientras que en la Escala de tendencias sociopáticas el grupo de alumnos de los diferentes conos que no han participado en actos violentos no muestran diferencias en sus puntuaciones medias entre ellos; los alumnos que conforman el grupo que ha participado en actos violentos, sí muestran diferencias entre los conos, obteniendo mayor puntaje en su puntuación media el Callao (14.57) frente al Cono Este (12.86) que obtuvo un puntaje medio menor.

Los resultados del análisis de varianza en las escalas de autojustificación, percepción del control parental y tendencia sociopática, de acuerdo al sexo, grupo e interacción sexo-grupo, nos muestra que las diferencias están marcadas por el sexo y el grupo en las tres escalas; obteniendo mayor media los varones que las mujeres y en cuanto al grupo la mayor puntuación fue obtenida por el grupo de alumnos que han participado en actos violentos 
respecto a los que no han participado en ellos.

Tabla 4

Análisis de Varianza entre el sexo, grupos y sexo-grupo

\begin{tabular}{|c|l|c|c|}
\hline ESCALAS & \multicolumn{1}{|c|}{ VARIABLE } & F & P \\
\hline \multirow{2}{*}{ I } & Sexo & 25.81 & $.000^{*}$ \\
& Grupo & 16.38 & $.000{ }^{*}$ \\
& Sexo-Grupo & 008 & .781 \\
\hline \multirow{3}{*}{ II } & Sexo & 21.59 & $.000^{*}$ \\
& Grupo & 5.79 & $.016^{*}$ \\
& Sexo-Grupo & 2.15 & .143 \\
\hline \multirow{3}{*}{ III } & Sexo & 20.83 & $.000^{*}$ \\
& Grupo & 7.73 & $.000^{*}$ \\
& Sexo-Grupo & 0.53 & .466 \\
\hline
\end{tabular}

\section{DISCUSIÓN}

- La participación de los grupos de alumnos en actos violentos, evaluados por las escalas de autojustificación, percepción del control parental y tendencia sociopática, evidencia que estos grupos mantienen un conjunto de comportamientos sociales que retroalimenta la escalada de actos violentos en las cuales toman parte. Del mismo modo se podría sustentar que aquellos grupos que no participan en actos violentos, se diferencian de los anteriores al percibirse y actuar alejándose de los grupos que generan y/o mantiene la violencia entre los estudiantes.

- Las diferencias entre los grupos que participan en actos violentos con relación a la autojustificación se presenta en mayor proporción que los grupos no participantes pues éstos no justifican la violencia juvenil.

- Los jóvenes que participan en actos violentos tienen mayor percepción de control parental, lo que aparentemente se contradeciría con la de los jóvenes que no participan en actos violentos, puesto que estos no poseen un grado apreciable de control parental. Sería necesario precisar los niveles y la orientación de dicho control parental, 
infiriéndose que a mayor percepción de rigidez en el control, mayor reacción hacia la violencia.

- La tendencia sociopática es mayor y significativa en los jóvenes que participan de los actos violentos pues no sólo lo hacen de modo pasivo sino de una manera activa, evidenciándose así sus propios rasgos de personalidad tendientes a desarrollar conductas antisociales.

- El grupo que ha participado en actos violentos marca la diferencia con relación a otros grupos en los distintos Conos de Lima Metropolitana, lo que evidencia que estos grupos en el ámbito de los Conos se perciben a sí mismos como determinantes de los conflictos violentos.

- La presencia de los grupos en la distribución geográfica por Conos en Lima Metropolitana nos permite determinar algunas características:

- Con relación a la autojustificación, tal como lo sustentamos en la primera conclusión, las diferencias son notables entre grupos que participan o no en actos violentos, no mostrándose dichas diferencias cuando se comparan los grupos en diferentes conos de Lima.

- Más bien es en la percepción del control de los padres para los grupos que no participan en actos violentos donde se evidencia un marcado énfasis en el Cercado de Lima con relación al cono Sur, en donde se notaría una mayor percepción de permisividad de los padres con respecto a los hijos.

- Por otro lado y también en cuanto a la percepción del control parental, para los grupos que participan en actos violentos, a los padres de familia del Callao se les percibe como más controladores que el resto de padres de los Conos, siendo el cono Este donde existiría menor control parental. Este hecho confirma la afirmación sustentada líneas arriba de mayor proclividad a la violencia.

- Es en la Provincia del Callao donde se hace más evidente la tendencia sociopática de los jóvenes que participan en actos violentos.

- No por conocida la evidencia de las diferencias de género en la participación en actos de violencia, se puede dejar de mencionar el predominio de actos violentos en los varones 
sobre las mujeres, tanto a nivel de los grupos de alumnos que han y no han participado en actos violentos como de los conos de Lima Metropolitana.

\section{CONCLUSIONES}

De los resultados y conclusiones de la presente investigación es evidente que se debe realizar acciones coordinadas entre los diferentes sectores para generar programas a diferentes niveles (prevención y asistencial) tomando en cuenta las actitudes y percepción de los propios alumnos de acuerdo a su experiencia y ubicación geográfica.

Deben estructurarse capacitaciones permanentes -por diferentes medios- a los Padres sobre "como deben manejar las conductas de sus hijos que participan en actos violentos" y "como pueden controlar su agresividad".

\section{REFERENCIAS BIBLIOGRÁFICAS}

Anicama, J. (1989). Análisis conductual de la violencia y la agresión. El analista de la conducta (Boletín SPAMC). XVII (1). 20-33.

Arenas, C. (1998). Los rostros escondidos y la acción social frente a la marginalidad: violencia juvenil de los 90. Revista de psicología de la UNMSM. II(1).93-106.

Cruzado, J. (1997). La violencia juvenil (folleto). Lima: Instituto de Defensa Legal.

Elizalde R. Y cols. (1997). Actitudes hacia la violencia en jóvenes universitarios. Revista de psicología de la UNMSM.I(1).107-128.

García L. (1995). Las pandillas juveniles. Boletín de post-grado de la Facultad de Psicología de la UNMSM. V (4-5).

Martínez M. Y Tong F. (1998). ¿Nacidos para ser salvajes? Identidad y violencia juvenil en los 90. Lima. CEAPAZ.

Masías, C. (1994). Efectos de un programa integral de sustitución de la agresión en un grupo de adolescentes hombres de alto riesgo entre 14 y 18 años de edad, de sectores tugurizados de Barrios altos. Tesis de Maestría. Lima UPSMP. 
Masías, C. y Anicama, J. (1994). Experiencias en la Aplicación de programas preventivos y de sustitución de la violencia. El Programa ART. En J. Anicama (Ed.). Drogas y violencia. Lima: CEDRO.

Moragues, M. (1989). Educar para el autogobierno. Formar actitudes de creatividad, criticidad, libertad y solidaridad en niños y adolescentes. Lima: Tarea.

Morales, J. F. (1994). Psicología social. Madrid: McGraw-Hill. Seco IX, Cap. 17. pp 466.

Montgomery, W. (1998). El Problema de la Violencia Juvenil: Análisis Teorético y de Programas de intervención conductual. Revista de Investigación en Psicología IPSI de la UNMSM. Vol. I (1) pp 133-152.

Myers, D. (1995). Psicología social. Cuarta edición. México: McGrawHill/Interamericana. pp. 390-391.

Orellana, O. y García L. (1996). Violencia y representaciones sociales en escolares. Revista peruana de psicología. I (1).26-36.

Tong, F. (1995). Los jóvenes pandilleros: entre el estigma y la epopeya (Folleto). Lima: Tarea.

Trathemberg, L. y otros (1995). Violencia juvenil: causas y alternativas (Mesa redonda). Lima: Congreso de la República del Perú.

Vallejos, J. y Montero. V. (1990). La psicología comunitaria en el Perú (II).

Psicología actual. II (6). 15-21.

Varona, S. (1991). Efectos de un programa para la sustitución de la agresión mediante el desarrollo de habilidades sociales y el autocontrol en menores de 11 - 15 años, en condiciones especialmente difíciles, institucionalizados del COMAIN. Tesis de Maestría: UPCH. 


\section{ANEXO 1}

Coeficiente de validez de cada uno de los ítems por el método Item-Test de la escala de

Autojustificación

\begin{tabular}{|c|c|c|c|c|c|}
\hline ITEMS & $\begin{array}{c}\text { coeficiente de } \\
\text { validez }\end{array}$ & ITEMS & $\begin{array}{c}\text { coeficiente de } \\
\text { validez }\end{array}$ & ITEMS & coeficiente de validez \\
\hline 1 & 0.288 & 12 & 0.3026 & 23 & 0.4119 \\
\hline 3 & 0.4408 & 15 & 0.3774 & 25 & 0.4519 \\
\hline 5 & 0.3212 & 16 & 0.3166 & 27 & 0.3626 \\
\hline 6 & 0.3224 & 17 & 0.3829 & 28 & 0.2819 \\
\hline 8 & 0.3645 & 20 & 0.3081 & 30 & 0.3593 \\
\hline 10 & 0.3623 & 21 & 0.4808 & & \\
\hline
\end{tabular}

Coeficiente de validez de cada uno de los ítems por el método Item-Test de percepción de control parental

\begin{tabular}{|c|c|c|c|c|c|}
\hline ITEMS & $\begin{array}{c}\text { coeficiente de } \\
\text { validez }\end{array}$ & ITEMS & coeficiente de validez & ITEMS & $\begin{array}{c}\text { coeficiente de } \\
\text { validez }\end{array}$ \\
\hline 7 & 0.4681 & 11 & 0.3342 & 18 & 0.2539 \\
\hline 9 & 2751 & 14 & 0.3247 & 26 & 0.3284 \\
\hline
\end{tabular}

Coeficiente de validez de cada uno de los ítems por el método Item-Test de la escala de tendencia sociopática

\begin{tabular}{|c|c|c|c|c|c|}
\hline ITEMS & $\begin{array}{c}\text { coeficiente de } \\
\text { validez }\end{array}$ & ITEMS & $\begin{array}{c}\text { coeficiente de } \\
\text { validez }\end{array}$ & ITEMS & coeficiente de validez \\
\hline 4 & 0.2218 & 22 & 0.1907 & 29 & 0.2855 \\
\hline 19 & 0.2367 & 24 & 0.303 & \multicolumn{2}{|c}{} \\
\cline { 1 - 4 }
\end{tabular}

Coeficiente de confiabilidad de las escalas por el método Alfa de Crombach

\begin{tabular}{|c|c|}
\hline Escalas & Coeficiente de Confiabilidad \\
\hline Autojustificación & 0.7757 \\
\hline Percepción del control parental & 0.5963 \\
\hline Tendencia sociopática & 0.4652 \\
\hline
\end{tabular}

